\title{
Norois
}

Environnement, aménagement, société

\section{Atlanpole : les fragilités d'une organisation spatiale et thématique}

Atlanpole: fragilities of a spatial and specialization organization

Jacques Fache

\section{OpenEdition}

1 Journals

\section{Édition électronique}

URL : https://journals.openedition.org/norois/1789

DOI : 10.4000/norois. 1789

ISBN : 978-2-7535-1548-2

ISSN : $1760-8546$

\section{Éditeur}

Presses universitaires de Rennes

\section{Édition imprimée}

Date de publication : 1 septembre 2006

Pagination : 25-37

ISBN : 978-2-7535-0342-7

ISSN : 0029-182X

\section{Référence électronique}

Jacques Fache, «Atlanpole : les fragilités d'une organisation spatiale et thématique », Norois [En ligne], 200 | 2006/3, mis en ligne le 12 décembre 2008, consulté le 14 janvier 2022. URL : http:// journals.openedition.org/norois/1789; DOI : https://doi.org/10.4000/norois.1789

\section{(c) Tous droits réservés}




\title{
AtLanpole :
}

\section{LES FRAGILITÉS D’UNE ORGANISATION SPATIALE ET THÉMATIQUE}

\author{
JaCQues FACHE \\ IGARUN - UNIVERSITÉ DE NANTES \\ LACQ, CESTAN, ESO, UMR 6590 - CNRS \\ jacques.fache@univ-nantes.fr
}

\begin{abstract}
RÉSUMÉ
Les territoires sont de plus en plus en concurrence. Les technopoles en sont une composante essentielle. Atlanpole développe une activité importante selon de multiples spécialités. Cette diversité, qui est à l'opposé de ce qui existe dans de multiples technopoles, s'avère peu favorable à l'émergence d'accumulations significatives d'activités dans un domaine d'excellence. La métropole nantaise est en retard sur ce qui se passe dans beaucoup d'autres technopoles, même implantés dans des villes plus petites. Cette stratégie risque donc de faire passer la ville à côté des réseaux technologiques qui l'intègreraient dans un réseau de métropole de rang national ou européen.
\end{abstract}

MoTS CLÉS : Biotechnologie - Industries informatique et électroniques - Nantes Région métropolitaine - Technopôle.

\section{ABSTRACT}

\section{Atlanpole: fragilities of a spatial and specialization organization}

Territories are more and more in competition. Science parks are an important part of it. Atlanpole develop important actions acording to various economic specialities. This diversity is a situation in opposition with what is observed in others science parks. The creation of meaningfull cumulative processes of high tech activities becomes very hard. The metropolis of Nantes is lagging behind a lot of others science parks, even if they are located in smaller cities. This lack of specialization strategy is dangerous, because Nantes is at risk of missing technological networks that would integrate it in a national or european metropolitan network.

KEY WORDS : Biotechnology - Computering and Electronic Industry - Metropolitan Region - Nantes, Science parks.

Les technopôles sont désormais une part intégrante, si ce n'est classique, de l'aménagement du territoire. Mode d'organisation et d'aménagement révolutionnaire des années 1960, il s'est ensuite diffusé au cours des années 1970 et 1980 (Benko, 1991) au point d'être désormais banalisé. Qui, parmi les grandes villes, n'a pas son technopôle, en France, en Europe ou en Amérique du Nord? Le processus est d'ailleurs loin de s'arrêter et touche désormais des villes moyennes. Troyes a lancé 
en 2000 son technopôle de l'Aube; Soissons vient de lancer celui de l'Aisne. Le succès de cette structure est aisément compréhensible. L'évolution de l'industrie, puis des services, place les pays les plus avancés en concurrence frontale et de plus en plus difficile avec les pays émergents, en particulier asiatiques, mais pas seulement. La porte de sortie de l'ensemble des activités se situe vers les plus hautes qualifications, en se lançant dans une infernale course aux compétences et savoir-faire afin de garder, autant que possible, une petite et fragile avance. Elle concerne aussi l'organisation des activités et des territoires, procurant potentiellement un avantage local dans un système global. Dans ce contexte, les technopôles ont été perçus, à tort ou à raison, comme l'outil permettant l'orientation de l'économie locale et régionale vers les activités à fort contenu de connaissances, ce que les anglo-saxons désignent désormais comme la «knowledge economy », et moteur de la croissance et du dynamisme ${ }^{1}$.

Nantes, en tant que capitale de région des Pays de la Loire et centre rayonnant sur l'ensemble du grand Ouest, n'a pas échappé à cette règle. La ville a tenté de négocier le virage de l'après chantiers navals par le développement de l'innovation technologique qui, selon les critères en vogue à l'époque, faisaient cruellement défaut à la cité des Ducs. Le technopôle de Nantes-Atlanpole est ainsi né la même année - signe fort - que la fermeture des derniers chantiers Dubigeon sur l'Ile de Nantes. 1987, c'est tard, voire très tard (trop ?), par rapport à de multiples zones existantes. Outre Sophia-Antipolis et Meylan, à la charnière des années 1960 et 1970, sont déjà lancés Lyon (fin des années 1970), Nancy (1978), Montpellier, Rennes, Toulouse, Marseille (milieu des années 1980) et bien d'autres. Mais la vraie question est sans doute ailleurs, dans la stratégie de développement du site qui transparaît par le développement des implantations depuis maintenant vingt ans. En effet, si les technopôles sont très différents, la finalité est la même partout : intégrer un système productif de biens ou services de haute technologie, ou y renforcer ses positions, afin de bâtir une base économique performante tirant la croissance et le développement local et régional. Les technopôles marquent à ce titre un tournant dans la logique d'aménagement local qui bascule vers une recherche de compétitivité territorialisée.

L'objet de notre réflexion sera l'orientation prise par le développement technopolitain. En effet, si la date d'apparition est un facteur de réussite d'un technopôle - plus le système est banalisé territorialement, moins il a de chances de faire la différence -, la spécialisation choisie en est un autre, tout aussi important. Le choix d'Atlanpole semble bien être aujourd'hui celui de la polyvalence et de la diversité. Cette orientation est à la fois originale et banale par rapport à ce qui se fait couramment dans le milieu. Dans la plupart des cas de grandes villes en effet, le technopôle est dédié à une activité, un domaine d'activité, ou un dénominateur commun fort qui le rend lisible et si possible performant, alors que la diversité l'emporte parmi les plus petites. Dès lors, la question se précise. Une stratégie de la diversité est-elle viable par rapport à la concurrence extérieure (Fache, 2005) ? Permet-elle de peser suffisamment pour se positionner de manière pertinente dans au moins un des domaines d'activité représentés?

Notre démarche sera donc en partie comparative. En effet, une présentation de la diversité des activités sera naturellement suivie d'une confrontation avec la situation observée dans d'autres technopôles qui n'ont pas nécessairement fait le choix de la polyvalence, mais qui, dans tel ou tel domaine, représentent un concurrent de choix pour Nantes. Le secteur des biotechnologies sera particulièrement intéressant à étudier. C'est tout d'abord un secteur majeur du XXI ${ }^{\mathrm{e}}$ siècle qui façonnera (et façonne d'ailleurs déjà) des régions de haute technologie. C'est ensuite un domaine qui pourrait être en phase avec un pôle d'excellence reconnu à Nantes, dans le domaine de la santé. C'est enfin un axe de communication fort par rapport auquel il est intéressant de faire la part des choses, entre mythe et réalité, en quelque sorte.

1. Le numéro spécial des Cahiers Français « croissance et innovation » dirigé par B. Ferrandon (2004) fait la synthèse des interactions entre innovation, croissance et développement, replaçant l'innovation au cœur de processus et théories économiques, mais développant aussi les stratégies d'acteurs à divers niveaux d'échelle. 


\section{Nantes, technopôle polyvalent, ou zone d'activité attrape-tout?}

D’un strict point de vue économique, le technopôle nantais se caractérise par une grande diversité (fig. 1). Une simple lecture des annuaires d'entreprises soutenues suffit à s'en convaincre : génie naval, biotechnologies, santé, NTIC ${ }^{2}$, matériaux, chimie, etc. Ce ne sont pas moins de six familles génériques qui sont présentes pour classer les entreprises (fig. 2).

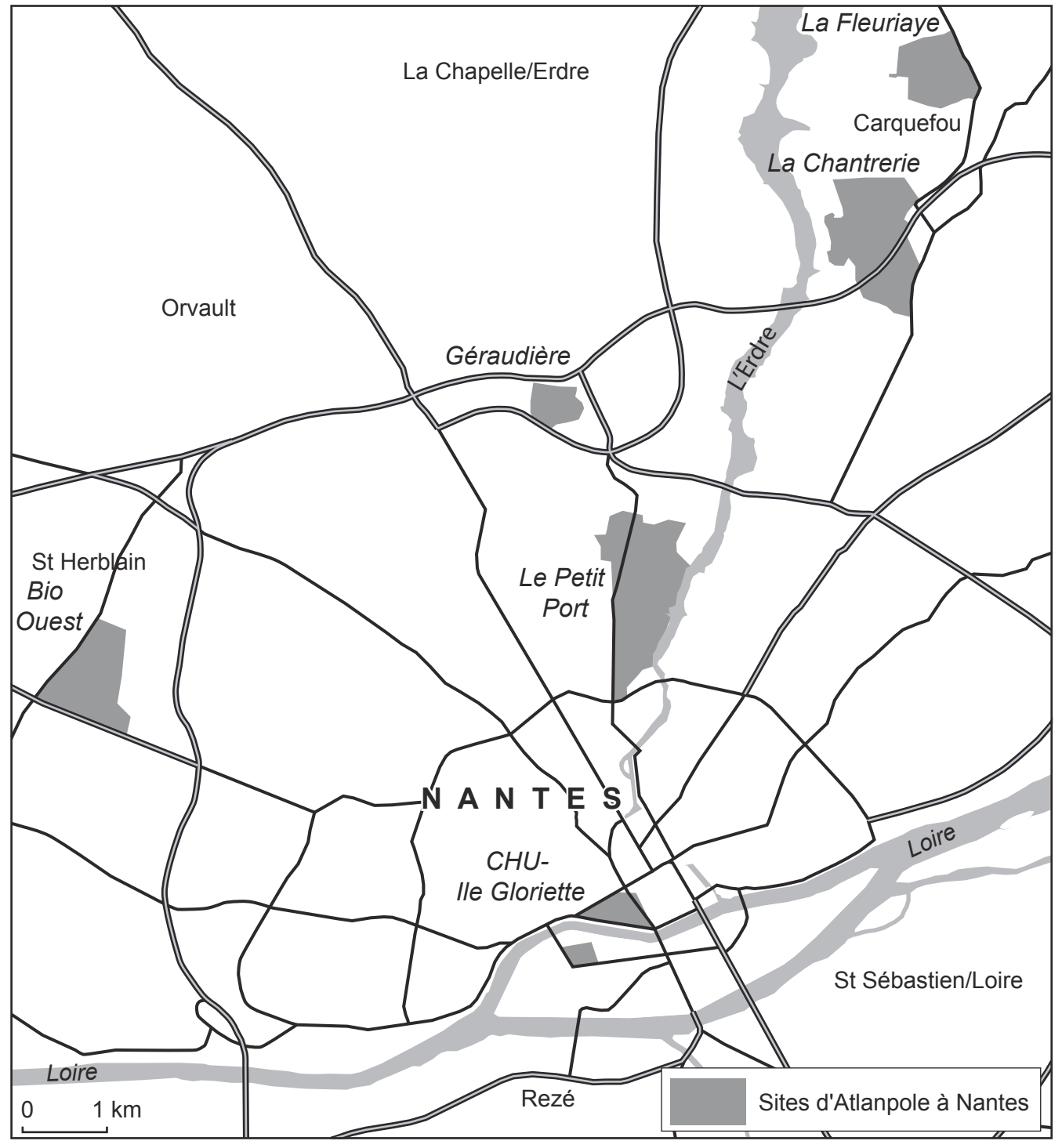

Figure 1 : Le technopôle de Nantes-Atlanpole - carte de localisation des sites Atlanpole technopole of Nantes - location map 


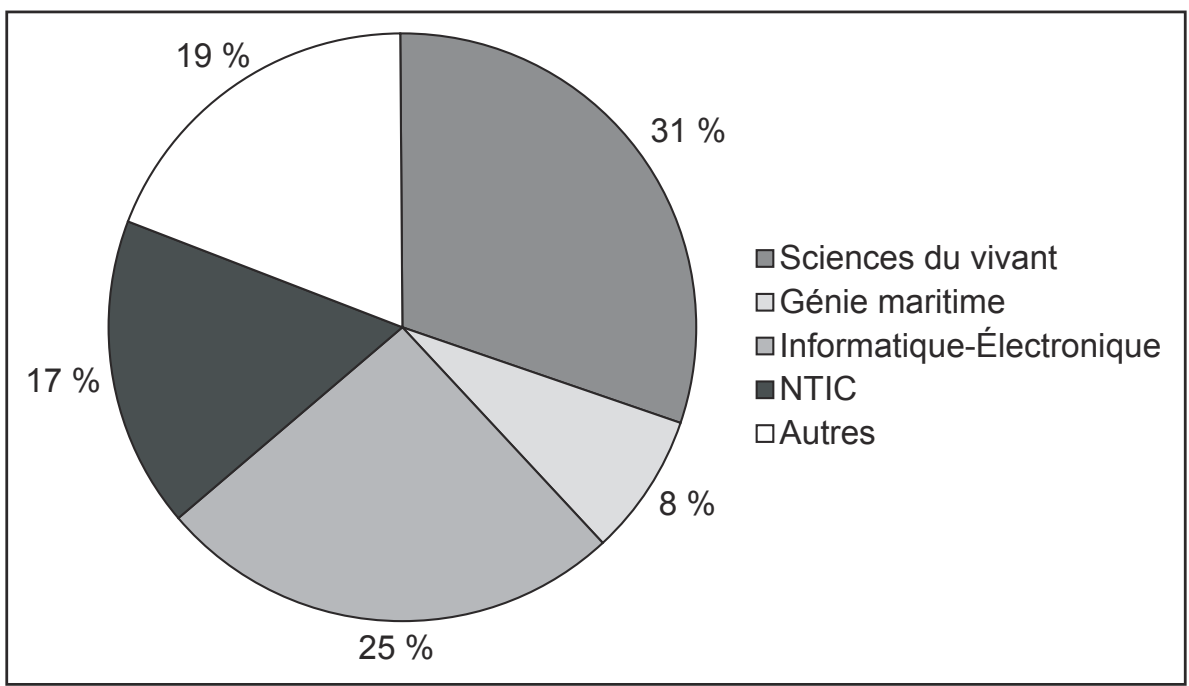

Figure 2 : Part (\%) des établissements d'Atlanpole selon les spécialités du technopôle (fin 2004) (end of 2004)

Part (\%) of plants located in Atlanpole according to specialities of the science park

Et encore faut-il considérer que chacune de ces familles est extrêmement large. Les technologies navales regroupent à la fois des entreprises industrielles de propulsion (DCN Propulsion), de l'informatique destinée à la navigation (Thalès Navigation), de l'étude et du test de carènes (Principia Marine), etc. Les sciences du vivant synthétisent les biotechnologies appliquées à l'agroalimentaire et à la santé. Or les compétences d'Eurofins (analyses de produits alimentaires) n'ont pas grand chose à voir avec Laennec Tek (test de molécules thérapeutiques sur le système cardio-vasculaire). La lecture de ces données laisse penser que malgré les apparences, Atlanpole est finalement assez spécialisée, puisque l'ensemble informatique-électronique-NTIC représente plus de $40 \%$ des entreprises et établissements implantés sur les sites. Les sciences du vivant représentent de leur côté plus de $30 \%$ des entreprises et établissements. Ces données sont fort trompeuses En effet, si ces grandes familles d'activités donnent une première idée des activités du site d'Atlanpole, une analyse plus fine des $\mathrm{NAF}^{3}$ présente une vision quelque peu différente (fig. 3).

La prédominance de deux familles d'activités est nettement moins évidente. L'informatique (NAF 721 à 724) ne représente plus que $26 \%$ des entreprises et de l'emploi ${ }^{4}$ du site, avec deux entreprises, Proservia et SNEDA, qui pèsent plus de $60 \%$ de l'emploi dans ce secteur. Les activités de recherche $(731 \mathrm{Z})$, totalement tournées vers les biotechnologies, tombent à $15,9 \%$, pour $7 \%$ de l'emploi. Les industries électriques et électroniques (IEE) ne comptent que trois entreprises, mais représentent plus du tiers des emplois du technopôle. Par contre, le secteur du service aux entreprises émerge clairement, comptant plus du tiers des entreprises pour 30,9\% de l'emploi.

3. NAF : Nomenclature des Activités Françaises. Classement des entreprises et établissements en fonction de leur activité principale. Les NAF 721 à 724 couvrent l'ensemble des activités de services, ingénierie, conseil, développement en informatique et réalisation de logiciels ; la NAF 732 couvre les activités de Recherche-Développement

4. Il s'agit de l'emploi dans les entreprises des sites d'Atlanpole. Si nous intégrons les personnels des établissements de formation et de recherche publics, c'est à dire la totalité de l'université ainsi que bon nombre des écoles d'ingénieurs (Mines, Centrale Nantes, etc.) et de centres de recherche (INRA, INSERM, IFREMER), ce pourcentage s'effondre. Par exemple, les seuls laboratoires de l'INSERM représentent plus de 600 chercheurs, soit plus de quatre fois les emplois des entreprises de R\&D en biotechnologies. 


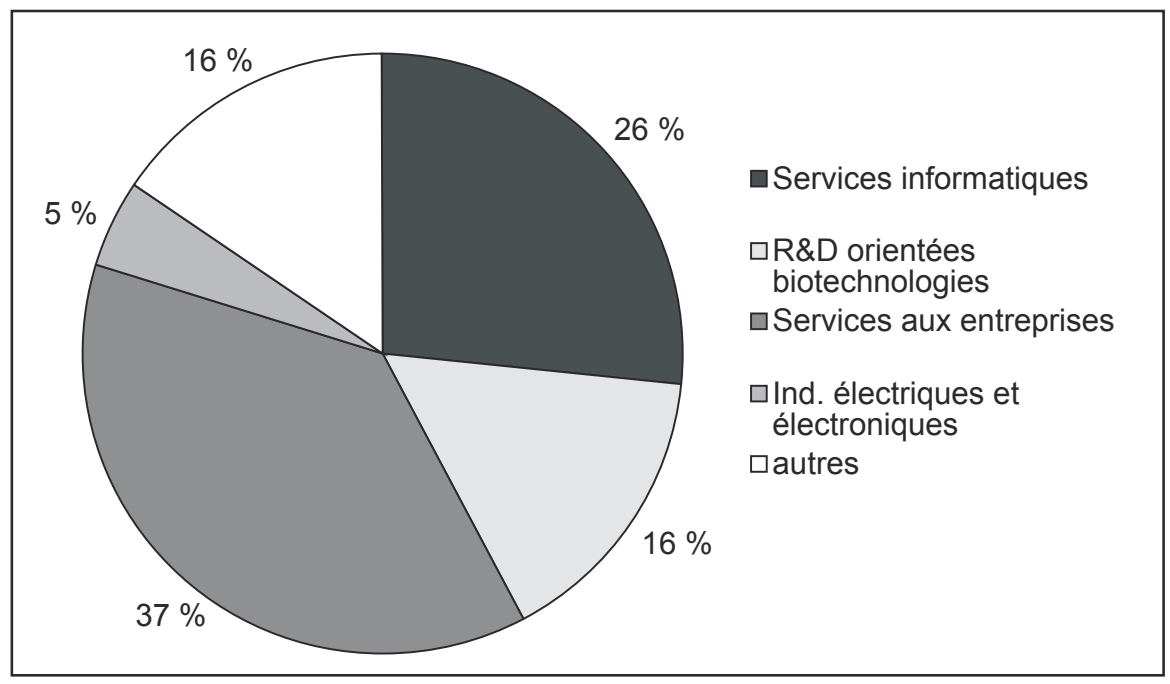

Figure 3 : Analyse par les NAF des activités atlanpolitaines (fin 2004) (\% des établissements) (\% of plants) Analysis acording to NAF of activities located in Atlanpole (end of 2004)

Le décalage entre les deux lectures peut aisément se comprendre. D’un côté, Atlanpole suit une logique de filière, regroupant les activités par grande famille thématique, pendant que l'INSEE les assemble par type extrêmement fin de produit ou de service rendu. Ainsi, les activités informatiques de haut niveau en viennent rapidement à être très différenciées, avec des passerelles fragiles d'un bord à l'autre, alimentant une série INSEE qui n'apprend rien sur les orientations professionnelles des entreprises... De ce fait, la modélisation numérique de carènes face aux contraintes océaniques n'a d'autres liens avec la santé que les langages utilisés pour la programmation.

Mais au-delà de ces questions de nomenclature, deux faits sont évidents. En premier lieu, l'éclatement géographique des types d'entreprises est extrêmement fort, et il est probable que pour nombre d'entres elles, le sentiment d'isolement doit exister. En effet, Atlanpole ne compte que 65 entreprises et établissements sur sites. Répartis dans toutes ces catégories, cela signifie que dans nombre de cas, les interlocuteurs sont peu nombreux, voire inexistants. L'intégration à ce stock d'entreprises atlanpolitaines non implantées sur site élargit le panel et améliore les choses puisqu'elles représentent deux fois plus d'établissements qu'Atlanpole en tant que tel. Mais au total, ce sont tout de même des effectifs bien modestes qui concernent par exemple les biotechnologies, avec 40 entreprises $\left(18,1 \%\right.$ ) pour 823 emplois ( $5 \%$ du total) ${ }^{5}$. Il est toujours très difficile de définir une masse critique, et ceci d'autant plus qu'elle dépend autant de critères quantitatifs que qualitatifs. Mais dans le cas présent, il est certain qu'Atlanpole et d'une manière générale Nantes ont un tissu d'entreprises bien limité dans les domaines de prédilection du technopôle. Le second élément est celui de la proximité géographique. Atlanpole compte 65 entreprises sur ses sept sites atlanpolitains. Le plus important, celui de La Chantrerie, compte 29 entreprises, dont 19 dans le bâtiment d'accueil du site. Biowest n'en regroupe que quatre. Il est donc évident que le faible nombre total d'activités dans chaque spécialité atlanpolitaine est éparpillé sur plusieurs sites. Ces minuscules grappes sont elles-mêmes parfois assez éloignées d'un tissu d'entreprises de la métropole qui, si elles sont atlanpolitaines, n’en sont pas moins diffuses. Or la proximité est un élément constitutif du technopôle et de son bon fonctionnement. Toutes les activités n’ont pas un

5. Le total d'emplois atlanpolitains souffre de la distorsion introduite par des mastodontes comme les Chantiers de l'Atlantique. Ceux-ci comptent plus de 4000 emplois qui, pour l'essentiel, ne relèvent tout de même pas de la haute technologie. Mais même en tenant compte de ces quelques entreprises, les biotechnologies restent nettement en deçà du seuil des $10 \%$. 
besoin équivalent de concentration pour développer leur innovation, mais pour certains secteurs innovants comme les NTIC, les biotechnologies ou les nanotechnologies, elle est indispensable, comme le montre le cas grenoblois (Paillard, 2004). C'est par une proximité géographique que les acteurs interagissent pleinement. G. Colletis et al. (1997), entre autres, développent l'idée d'un ancrage territorial par cette proximité et ces liens non sécables technologiquement. Plus généralement, E. Leamer et M. Storper (2005) affirment le caractère indispensable d'une proximité géographique pour la transmission de «messages complexes » dont dépend de plus en plus l'économie. Même s'il ne faut pas en faire un facteur de localisation (Grosetti et Bès, 2002), la proximité fournit des « externalités technopolitaines ou de milieu » qui permettent d'envisager une persistance sur le moyen et long terme (Decoster et al., 2004). C'est aussi dans des territoires bien identifiés que se constitue une sorte de creuset développant une proximité culturelle des acteurs qui en fait toute l'originalité et la performance ${ }^{6}$. Cet éparpillement est donc assez peu conforme avec les multiples observations des géographes et économistes. Il n'est même pas compensé par une spécificité de chaque site d'Atlanpole. Ainsi, les quinze entreprises relevant des biotechnologies se répartissent sur quatre sites. Il en est de même pour les quatorze entreprises de NTIC. Dans ces conditions, il est extrêmement difficile de susciter l'émergence d'un milieu local, malgré les efforts d'animation du site par l'équipe.

Une étude temporelle des secteurs d'activités nantais met en valeur une certaine réactivité face à la conjoncture (tableau 1).

\begin{tabular}{|c|c|c|c|c|c|}
\hline & & & 20 & 20 & 2005 \\
\hline Biosciences & Atlanpolitaines & 24 & 29 & 41 & 58 \\
\hline Dioserentes & dont technopole & 8 & 11 & 17 & 19 \\
\hline & $\%$ & 33,3 & 37,9 & 41,5 & 32,8 \\
\hline Informatique & Atlanpolitaines & 30 & 34 & 35 & 39 \\
\hline Electronique & dont technopole & 18 & 18 & 14 & 16 \\
\hline & $\%$ & 60,0 & 52,9 & 40,0 & 41,0 \\
\hline NTIC & Atlanpolitaines & 28 & 29 & 39 & 30 \\
\hline IVIIC & dont technopole & 16 & 14 & 18 & 9 \\
\hline & $\%$ & 57,1 & 48,3 & 46,2 & 30,0 \\
\hline NTIC+IF & Atlanpolitaines & 0 & 0 & 0 & 25 \\
\hline & dont technopole & 0 & 0 & 0 & $\begin{array}{l}5 \\
200\end{array}$ \\
\hline
\end{tabular}

Tableau 1 : Évolution du nombre des entreprises technopolitaines et associées (source : Annuaires Atlanpole - [www.atlanpole.fr])

Evolution of firms located in the science park and associated ones

Il est périlleux de tirer des conclusions définitives sur une si courte période. Cependant, il apparaît clairement trois éléments d'analyse. Tout d'abord, les entreprises relevant des biosciences, pour reprendre un terme générique d'Atlanpole, sont en très forte croissance globale, avec à la clé une montée régulière de ces entreprises sur les sites atlanpolitains puisqu'elles se sont multipliées par plus de deux. Dans les domaines de l'informatique-électronique et des NTIC, la croissance est moindre jusqu'à la fin 2004-début 2005, date à laquelle le chiffre des entreprises atlanpolitaines repart à la hausse. Sur les sites du technopôle, ces activités restent d'une grande stabilité, voire décroissent. Second élément, la croissance des entreprises atlanpolitaines s'effectue essentiellement par rattachement d'éléments extérieurs aux sites du technopôle, dont les bâtiments

6. M. Bernardy de Sigoyer et P. Boisgontier ont très finement analysé ces rapports de proximité et les « effets de milieu » dans leurs ouvrages de 1988 et 1996 sur Grenoble qui constituent deux des analyses les plus fines qui soient du fonctionnement interne d'un technopôle, Meylan. 
d'accueil sont désormais pleins ou quasiment remplis, et les sites largement occupés, amenant au développement de zones nouvelles, mais non technopolitaines, comme La Chantrerie II. La part des entreprises sur sites baisse donc régulièrement en valeur relative. Ceci amène donc le dernier point : les sites atlanpolitains ne semblent pas en mesure, au moins pour des raisons physiques de disponibilités de terrains et de locaux, de constituer des grappes d'entreprises importantes et doivent se rabattre sur une croissance externe diffuse, ce qui ramène à la question de la proximité déjà évoquée.

Ces variations soulèvent de nombreuses questions, à commencer par celle de la stratégie. En effet, le technopôle a clairement su utiliser la poussée de l'électronique et surtout des NTIC, puis des biotechnologies qui est en cours. Surfer sur des vagues porteuses type NTIC et biotechs peut sembler une bonne stratégie. La structure est à peu près sûre, dans un contexte porteur, de récupérer des implantations et une partie de la dynamique générale. Mais un technopôle est-il là pour faire ce que tout le monde fait et au même moment, si possible mieux, ou pour anticiper et faire avec dix ans d'avance ce que tout le monde fera ensuite? Car débuter un cycle très tôt, si possible le premier, est la seule vraie garantie sinon du succès, du moins de l'avantage que l'on en tirera, à charge ensuite de le faire fructifier et de le maintenir dans le temps ${ }^{7}$. Accrocher des cycles de produits en cours, c'est tout d'abord accepter systématiquement de partir dans la course avec un retard conséquent sur les leaders, et par là même se positionner dans un rôle de second - peut-être brillant - mais de second tout de même! Quand l'ambition affichée est de devenir une métropole européenne, c'est un peu gênant. En effet, les grandes métropoles du continent se situent très souvent dans un rôle d'initiatrices à un moment donné. Mais le positionnement sur des cycles en cours, c'est aussi se situer dans une perspective quantitative et qualitative moyenne, voire médiocre. Les activités nouvelles connaissant une forte croissance commencent en effet à se diffuser et donc à se répartir sur de multiples sites technopolitains et/ou métropolitains qui, se concurrençant âprement, limitent de fait la quantité finale d'entreprises et d'établissements créés. Quant aux compétences de pointe, comment dans ces conditions s'approprier celles des centres initiaux? En d'autres termes, et pour être synthétique, où se situe dans ce cadre l'avantage technologique et économique pour le territoire d'accueil du technopôle? Dans le cas qui nous intéresse, la structure a-t-elle fait autre chose que d'accompagner une dynamique globale en cours impulsée par d'autres? Or l'un des paramètres de la constitution de technopôles est l'anticipation. C. Genet (1997) identifie ainsi trois variables clé à la formation d'un biotechnopôle : les externalités technologiques localisées, la dynamique historique et les dynamiques liées aux anticipations d'agents.

Le problème a très récemment été dévoilé par la politique des «pôles de compétitivité » dont la liste a été annoncée en juillet 2005. Cette stratégie de développement visant à renforcer quelques locomotives puissantes pour tirer la croissance et le développement de la France devait initialement identifier une quinzaine de pôles performants, correspondant aux « six pôles mondiaux » et « neuf pôles à vocation mondiale » du choix ministériel final. À l'arrivée, ce sont 67 pôles qui ont été identifiés, traduisant là un souci politique d'élargir le champ d'application de cette politique afin d'en limiter les frustrations locales et régionales. Dans ce contexte, où se situe Nantes? Loin, très loin des pôles leaders. Aucun «pôle mondial » ni « à vocation mondiale », pas plus que de «pôles interrégionaux » n'apparaissent. C'est au titre de «pôles mono-régionaux » que Nantes apparaît, avec les pôles Biothérapies, Génie Civil et EMC2. Dans leur ouvrage récent, N. Jacquet et D. Darmon (2005) extraient sept pôles nationaux en plus des quinze mondiaux. Les pôles nantais n'y figurent pas. C'est la seule des dix plus grandes villes française dans ce cas de figure. Et même dans ces cas là, ce n'est pas un processus d'accumulation métropolitain qui permet à la ville d'émerger, mais des assemblages régionaux composites regroupant des compétences variées provenant d'historiques de localisation hétéroclites. Ainsi en est-il d'EMC2. Les quatre donneurs

7. Il est tout à fait possible de transposer l'étude de D. Freeman (1985) sur la rente d'adoption de l'innovation. Le premier tire le maximum de l'adoption. Puis les suivants entrent dans une phase de rentabilité décroissante, jusqu'à ce que l'innovation ne rapporte plus d'avantage substantiel au nouvel adoptant. 
d'ordres régionaux structurant le projet appartiennent à des contextes fort différents. Airbus se situe dans la lignée de Bréguet, lui-même repreneur de l'activité industrielle aéronautique de Dubigeon, qui avait vainement tenté dans les années 1920 de se diversifier. Les chantiers de l'Atlantique et DCN Propulsion constituent les vestiges de la construction navale, constamment sur le fil du rasoir puisque les commandes se comptent en unités, et entraînent le même psychodrame régulier autour de la poursuite de l'activité à chaque fin de chantier. Bénéteau enfin, basé en Vendée, est le fruit de la progression régulière (en tendance) de la plaisance mondiale. Derrière tout cela, point d'accumulation métropolitaine, pas plus que d'émergence liée à un gisement de compétences de haute technologie par exemple. Quant aux biothérapies, l'accent est clairement mis sur les compétences académiques nantaises, en d'autres termes celles de l'INSERM du CHU, du cancéropôle et de l'hôpital Laënnec. Mais au-delà, où sont les firmes liées à la santé ayant poussé sur ce terreau, ou s'étant rapprochées de ce pôle d'excellence? Il en existe, certes, et qui ont d'ailleurs été en partie incubées par Atlanpole. Mais cela reste très limité, en partie pour des raisons de taille et de relative jeunesse de la montée en puissance du pôle santé nantais.

Ces réalités interrogent la dynamique technopolitaine. En premier lieu, il est évident qu'en vingt ans, il n'y a eu aucune accumulation spécialisée suffisamment significative pour que Nantes émergeât ne fût-ce qu'à un niveau national. Ensuite se pose le problème du risque de décrochage. Si les «pôles mondiaux » et à « vocation mondiale » sortent vraiment renforcés par l'action publique, une métropole régionale comme Nantes ne pourra espérer relever le gant qu'avec une stratégie d'accumulation territorialisée claire et lisible. Pour le moment les forces en présence restent bien modestes par rapport à la concurrence.

\section{Polyvalence ou spécialisation?}

Les territoires, à quelque niveau d'échelle que ce soit, sont désormais officiellement lancés dans une concurrence acharnée les uns par rapport aux autres. C'est l'aboutissement logique d'une volonté de décentralisation et de territorialisation de l'action économique. En responsabilisant les politiques de tous niveaux d'échelle géographique par rapport au développement, entre autres économique, le législateur les a implicitement ou explicitement mis dans une situation de comparaison et de recherche d'avantages compétitifs. Les technopôles relèvent souvent de cette recherche, et doivent donc être étudiés non pas à l'aune de leurs résultats bruts, mais à celle de leurs résultats relatifs. La première interrogation concerne la polyvalence d'Atlanpole. Est-elle une pratique courante?

\section{LA SPÉCIALISATION : DES DESTINS VARIÉS ${ }^{8}$}

La spécialisation des technopôles est une réalité variant selon la taille du technopôle et son histoire. Les technopôles historiques, Sophia-Antipolis et la ZIRST ${ }^{9}$ de Meylan (récemment devenue Innovallée), ont aboutit à une spécialisation par des chemins variés. Meylan s'est en effet appuyé dès le départ sur ses activités de base, l'électronique et informatique, pendant que Sophia-Antipolis attrapait un peu tout ce qui voulait s'implanter tant que c'était de la recherche, pour se recentrer dans un second temps sur deux domaines plus précis ${ }^{10}$ (les sciences de la vie et les sciences de l'information). Ces spécialités désormais acquises restent fortes. Innovallée compte ainsi plus d'une centaines d'entreprises de services informatiques (sur 270), s'orientant tant vers le logiciel très spécialisé que vers l'architecture de réseau. Les nouvelles orientations d'Innovallée vers la

8. Sauf indication contraire, les nombres d'entreprises et d'employés proviennent des organismes gérant les technopôles, via leurs sites ou leurs lettres d'information.

9. ZIRST : Zone pour l'Innovation et les Réalisations Scientifiques et Techniques.

10. M. Quéré (2002) identifie les deux phases stratégiques et les évolutions de jeux d'acteurs dans les temps. A. Grondeau (2005) oppose justement Sophia-Antipolis à son illustre prédécesseur, La Silicon Valley, quant à ses origines, politiques et volontaristes. La même différence l’oppose de fait à Innovallée. 
microélectronique et les nanotechnologies découlent de ce passé grenoblois et de l'évolution des produits informatiques, et donnent une très forte cohérence à l'ensemble derrière une diversité apparente (4 spécialités revendiquées). Désormais, le matériel informatique est pour l'essentiel une production banalisée, de même que de nombreuses activités logicielles. Par contre, la microélectronique reste porteuse et attractive, avec des implantations durables comme Motorola en 2002. Les grands acteurs s'allient pour constituer des groupes de recherche performants. STMicroelectronics, Philips et Motorola développent la coopération Crolles II autour des puces miniaturisées à l'échelle subatomique (Paillard, 2004). Sophia-Antipolis est dans une situation analogue. Désormais, son fichier d'entreprises n'identifie plus un secteur de l'informatique, mais des sciences de l'information. Cela pourrait passer pour un terme attrape-tout, mais cela correspond bien à la réalité de l'évolution de l'informatique dans son ensemble, y compris pour Nantes. Ce sont donc 336 entreprises du site qui s'y consacrent, pour un total de 12328 emplois ${ }^{11}$. Ces chiffres sont singulièrement élevés, et traduisent pour une part la stratégie de Sophia-Antipolis, misant dès le départ sur des implantations de grandes firmes internationales et accueillant actuellement Amadeus, Equant France, Infineon Technologies, Philips Semiconductors, etc. Les technopôles de grandes métropoles françaises ont aussi opté pour des spécialisations d'autant plus naturelles que les secteurs d'activités étaient déjà présent dans la ville. Dans l'agglomération parisienne, les pôles technologiques sont multiples, puissants, et peuvent sans grande difficultés se targuer d'un grand nombre de références très spécialisées. Le terme de technopole n'est d'ailleurs même plus employé, délaissé pour des appellations plus évocatrices; le Génopole $囚$ d’Evry, ou Paris Biotech dans le XIVe arrondissement (donc deux pôles santé pour Paris là où tant de villes ont du mal à en monter un, avec deux « hyper » spécialités, la génétique pour l'un, le médicament et la robotique médicale pour l'autre). Lyon affiche une spécialité dans les biotechnologies avec plus de 150 entreprises réparties sur les sites de La Doua et de Gerland ${ }^{12}$. Des villes de taille modeste peuvent abriter un technopôle très spécialisé et d’importance. Mais cela dépend souvent de contextes politico-économiques favorables permettant d'acquérir une spécificité forte. Lannion est ainsi devenu le support d'Anticipa, technopôle du Trégor, fort de dizaines d'entreprises de télécommunication dont les plus grands noms mondiaux. Cet exemple s'est appuyé sur le développement exceptionnel des télécommunications en Bretagne sous l'impulsion de l'Etat dans les années 1960. Mais il reste exceptionnel.

\section{LA SPÉCIALISATION : UNE RÈGLE GÉNÉRALE POUR LES GRANDES VILLES ET MÉTROPOLES}

Au total, sur les 47 technopôles labellisés par l'IASP ${ }^{13}$, huit n'ont qu'un secteur d'intervention revendiqué, et près de la moitié $(46 \%)$ se limitent à trois. Mais si l'on va au-delà des simples effets d'affichage, les spécialisations sont souvent plus réduites que ne le laisse supposer la communication technopolitaine. Innovallée affiche quatre domaines, pour un largement dominant;

11. Sophia-Antipolis compte 1333 entreprises et organismes dans son parc pour 26942 emplois. Mais bon nombre de ces entreprises et emplois sont liés à des activités de services banals. L'emploi dans les secteurs relevant des hautes technologies et de la recherche représente 14842 emplois pour 410 entreprises, et l'enseignement supérieur-recherche 2465 personnes pour 54 entités. L'informatique représente donc $83 \%$ de l’emploi de haute technologie du site (source : Sophia-Antipolis). Même si ce chiffre est à nuancer, car nous y retrouvons aussi bien de la recherche sur les semi-conducteurs que de l'architecture de réseau ou du logiciel professionnel, la spécialisation reste indéniable, et la cohérence dans l'évolution forte.

12. M. Bonneville (1997) analyse le développement de la biochimie et le poids de familles comme Mérieux, le poids de la recherche. Il montre aussi l'utilisation du parc scientifique de Gerland comme outil de rénovation urbaine et d'aménagement autour d'anciennes implantations Mérieux.

13. L'inflation et le galvaudage du terme de technopôle a amené les gestionnaires des technopôles historiques et puissants à se regrouper dans une association, l'International Association of Science Park (IASP) afin de fixer des critères définissant le technopôle et permettant de le labelliser, tout comme les Centres Européens d'Entreprises et d'Innovation (CEEI) sont labellisés par l'Union Européenne ou les incubateurs le sont selon l'appel à projet de 1999 du ministère. Mais cela n'empêche pas qui le veut de s'autoproclamer technopôle, comme c'est le cas du Futuroscope de Poitiers, de Micropolis à Gap ou du Technopôle du Madrillet à Rouen. Les créations très récentes sont aussi parfois trop jeunes pour remplir toutes les conditions de labellisation (Le Madrillet, ou encore le technopôle de l'Aisne, à Soisson). 
Rennes en affiche quatre aussi, pour une forte spécialité en informatique-télécommunications; Besançon, avec Thémis, décline une multitude de secteurs d'intervention, convergeant en réalité vers un dénominateur commun, les microtechniques dans tous les domaines. Dans certains cas, la diversité doit être vue avec nuance. Ainsi, Montpellier revendique six domaines d'action, mais ceux-ci correspondent à une réalité géo-économique et scientifique de la ville : l'existence de points forts scientifiques déclinés en pôles spécialisés que le technopôle fédère. Certains de ces pôles sont d'ailleurs cousins : biotechnologies, biopharmacie, instrumentation médicale.

Nantes fait partie des onze technopôles labellisés (soit moins de $25 \%$ du total) qui revendiquent au moins cinq domaines d'action, et qui correspondent réellement à des secteurs différenciés et non complémentaires. Les cas de technopôles non spécialisés relèvent souvent du même profil. La ville-support représente une métropole à rayonnement régional ou une petite ville dont le passé industriel et tertiaire l'a coupé des développements récents des activités dites « de haute technologie », jugées à tort ou à raison comme des secteurs porteurs et d'avenir, notamment en termes d'emplois (Mulhouse, Nantes, Caen, Toulon, etc.). Dès lors, le technopôle représente un outil de valorisation suivant deux axes, déclinés de multiples manières. Il s'agit tout d'abord de valoriser, lorsqu'elle existe, la part d'innovation des activités traditionnelles, et d'opérer une reconversion par le haut. Le Mans et Mulhouse développent une spécialité mécanique, Annecy les équipements industriels, Nantes le génie maritime et technologies navales. Cette stratégie est louable, mais concerne des activités qui n'ont pas nécessairement besoin d'une hyperconcentration technopolitaine. Mais il faut aussi adjoindre un développement des activités porteuses, ou supposées telles pour l'avenir, parfois déjà présentes à l'état embryonnaire, mais aussi parfois complètement absentes. Dans ce domaine, les biotechnologies se taillent la part du lion, après que les NTIC eussent fait de même à la fin des années 1990. Sur les onze pôles polyvalents, six revendiquent un développement des «biotechnologies » ou «biosciences », auxquelles il faut rajouter trois «santé » ou « médecine » et cinq " agroalimentaire». Les NTIC concernent huit technopôles sur onze. Le souci de miser sur ces domaines est logique. Mais parmi ces technopoles, bien peu ont une réelle notoriété, et encore moins une attractivité forte sur leurs secteurs d'activité affichés. Ce n'est d'ailleurs pas un problème rédhibitoire si la ville a un réservoir d'entrepreneurs-innovateurs capable de répondre présent et de créer de l'activité. Mais dans ce cas de figure, le chemin est long jusqu'à une reconnaissance internationale ou même simplement nationale, le temps en fait d'accumuler quantitativement et qualitativement afin de constituer ce que d'aucun qualifie aujourd'hui de pôle de compétitivité.

\section{NANTES EN DÉCALAGE}

Atlanpole correspond assez bien à ce profil. Son histoire économique ne prédispose pas particulièrement la ville à développer des activités relevant des secteurs de pointe. Son rayonnement n'est pas suffisamment fort - ni sa spécialité suffisamment affichée - pour attirer des firmes du secteur. Les entreprises comme Atmel (ex Matra) ou Alcatel préexistaient au technopôle et ne se sont pas implantées à Nantes pour son tissu de haute technologie. De fait, la grande majorité des firmes atlanpolitaines est constituée soit de firmes incubées par la structure, soit d'entreprises préexistantes qui se sont associées à la dynamique et aux échanges qu'elle permettait. Mais pour le moment, les « success stories » à la nantaise sont assez rares, non pas sur le plan scientifique puisque de nombreuses entreprises sont de fait à la pointe de leur secteur, mais quantitatif. L'essentiel reste des microentreprises. Le seul cas ressortant est celui d'Eurofins, entreprise incubée en 1987, qui est passée de 12 employés à plus de 2000, dont environ 150 sur Nantes, avec des implantations européennes et étatsuniennes.

Cette situation est assez exceptionnelle pour une ville de cette taille. En effet, parmi les technopôles polyvalents, un seul est aussi le fait d'une métropole française importante : Bordeaux Technopole. À l'évidence, le décalage est important, et pour une ville dont le potentiel de départ n'est pas particulièrement puissant, il pose la question de la stratégie de développement tous azimuts et de la dispersion des efforts. L'équipe atlanpolitaine labourant de multiples terres favorise sans 
contestation possible l'émergence de nouvelles activités. Mais pour quel résultat final? Ou plus exactement pour quelle accumulation?

\section{Le technopôle face à ses concurrents}

Cette simple réalité stratégique soulève bien des problèmes de concurrence. En effet, Atlanpole ne peut être jugé sur un critère global de plus de 200 entreprises atlanpolitaines, chiffre trompeur, mais sur une juxtaposition de plusieurs sous-ensembles de quelques dizaines d'unités dans le meilleur des cas. La comparaison quantitative est donc beaucoup plus difficile à soutenir.

Atlanpole est en situation délicate sur le marché des technopôles. En effet, dans la plupart des domaines de spécialité, Nantes n'est qu'un site parmi beaucoup d'autres. Reprenons deux des cinq spécialités nantaises les plus importantes.

Les biotechnologies sont aujourd'hui partout, du moins sur le papier. Pas moins de 25 technopôles s'affichent dans ce domaine, avec des poids lourds historiques pour lesquels la structure ne fait que valoriser un existant riche (Lyon, Paris...). Il faut adjoindre à cela des concentrations scientifico-industrielles qui sont de type technopolitain sans prendre la peine d'en porter le nom. Les pôles parisiens et lyonnais sont une évidence, et ont servi de support à trois pôles de compétitivité mondiaux ou à vocation mondiale (Chesnais et Pijaudier-Cabot, 2005), dont deux pour Lyon ${ }^{14}$. Mais les pôles suivants sont loin d'être ridicules. Eurasanté à Lille regroupe plus de 80 entreprises sur site, et gère un réseau de 600 entreprises régionales. Montpellier joue sur sa tradition universitaire en médecine. Strasbourg en fait l'un de ces deux axes majeurs, et s'appuie sur une base économico-scientifique bien plus puissante que ne le laisserait supposer la taille de la ville. Elle compte plus de 200 laboratoires relevant du CNRS ou de l'INSERM, avec 3600 chercheurs. Il faut y rajouter le contexte industriel, avec des géants de la pharmacie (Bayer Polysan, Lilly France, Squibb, Roche, Synthélabo, etc.) et la proximité de Bâle, siège de Novartis. (Kleinsmager, 1999). Certains pôles sont émergents et sont susceptibles d'utiliser une dynamique globale déjà éprouvée ailleurs. Le pôle science-santé/chimie fine de SophiaAntipolis compte déjà 45 entreprises pour 2154 emplois ${ }^{15}$, avec quelques grands noms tels Rohm et Haas France, Galderma R\&D, Allergan France, Bayer Cropscience, etc. L'attractivité qui a joué en informatique est peut-être généralisable. Innovallée s'affirme avec 19 entreprises sur site.

Nantes ne compte que 45 entreprises atlanpolitaines, en regroupant des biotechnologiques ${ }^{16}$ relevant de la santé, du règne végétal et animal, ce qui ne fait pas nécessairement une cohérence. Techniquement, cela dénote d'un investissement fort au niveau de la création d'entreprises, puisque l'essentiel a été suscité à partir du milieu local. Mais malgré tout, cela place la ville très loin des meilleurs, malgré l'existence de pôles d'excellence universitaires dans le domaine de la santé, représentés entre autres par le Cancéropole et des unités de l'INSERM au CHU, en plein développement depuis plus de vingt ans. Les locomotives mondiales ou européennes sont rares. Bristol-Myers-Squibb (50 emplois) est présent à Saint-Nazaire depuis près de dix ans, et Eurofins (plus de 2000 emplois, dont environ 150 à Nantes) est le fruit d'une incubation réussie. Or dans tous les cas de figure, les grandes firmes sont des acteurs incontournables des biotechnologies, à la fois par leur potentiel interne, mais aussi par les contractualisations de recherche et l'essaimage, comme l'ont montré $\mathrm{A}$. Ferguène et F. Trimeche (2005) dans le cas de Lyon. Dès lors, il est très difficile pour Nantes de soutenir la comparaison et de pouvoir s'afficher dans ce domaine, face aux autres pôles identifiés.

Dans le domaine des NTIC et de l'informatique, le problème nantais est comparable. D'un point de vue comptable, Atlanpole compte 55 entreprises classées dans les NTIC (14 sur les sites d'Atlanpole) pour un effectif total de 1646 employés (587 sur Atlanpole). Dans ce domaine, la concurrence est peut-être encore plus lourde que pour les biotechnologies. 27 technopôles sur

14. Paris a dû sélectionner les projets présentés afin de ne pas tout rafler ( 14 dossiers déposés, soit moins que Poitou-Charente). Il n'y a ainsi aucun pôle mondial parisien en aéronautique, domaine pour lequel la métropole est leader.

15. Données de mars 2005.

16. Annuaire des entreprises atlanpolitaines, 2005. 
47 se placent en effet sur ce secteur, auxquels il faut rajouter deux technopôles revendiquant des télécommunications, chevauchant largement les questions de réseaux. Certes, tous ne se valent pas. Mais les poids lourds sont nombreux. En effet, les technopôles historiques sont partis très souvent de l'informatique, et sont de ce fait toujours très développés. Sophia-Antipolis et Innovallée constituent désormais des pôles puissants qui ont évolué vers les réseaux et les télécommunications (création d'un club Telecom Valley à Sophia, fédérant des entreprises du technopôle et hors technopole comme Aérospatiale, IBM et Texas Instrument [Quéré, 2002]). Mais d'autres pôles se situent bien sûr sur le même créneau : Paris, avec la concentration de Paris Sud tournant autour du complexe militaro-industriel, mais aussi le cyber-district du Silicon Sentier non labellisé technopôle mais qui y fait largement penser à certains égards ${ }^{17}$; Marseille, avec son site technopolitain de Luminy; Toulouse, avec les sites de haute technologie du Sicoval. Même des villes de plus petite taille sont mieux placées. Rennes-Atalante et Lannion retirent tout le bénéfice de la politique de développement des télécommunications dans l'Ouest. Si les usines s'en retirent, les laboratoires restent et même se renforcent. À chaque fois, ces pôles constituent l'ossature de systèmes moteurs, là encore reconnus en termes de pôles de compétitivité ${ }^{18}$. De ce fait, le développement des NTIC à Atlanpole semble bien plus relever de l'accompagnement du développement normal d'une métropole régionale que d'un surplus de dynamisme.

\section{Conclusion}

Au total, si la masse des entreprises atlanpolitaines est loin d'être négligeable, il est évident que le dispositif de polyvalence entraîne une dissémination sectorielle et spatiale des activités qui gêne la constitution de pôles d'accumulation qui feraient émerger la métropole sur une scène ou une autre. C'est particulièrement vrai pour les secteurs qui sont les plus en pointe et qui nécessitent, au moins en phase initiale, une concentration géographique des acteurs institutionnels et entrepreneuriaux. Les masses d'entreprises en jeu sont trop faibles, et si la dimension innovante des entreprises nantaises en vaut largement d'autres, il est difficile de concurrencer d'autres pôles plus structurés et plus massifs, y compris dans des villes de plus petite dimension que Nantes.

La jeunesse et l'histoire nantaises expliquent en partie cet état de fait, mais pas complètement. Cette stratégie est lourde de conséquences lorsque passe le train de certaines opérations d'aménagement comme celle des «pôles de compétitivité ». Il ne faut certes pas exagérer son importance financière : il s'agit de 1,5 milliard d'euros sur trois ans ${ }^{19}$, soit l'équivalent annuel de Hong-Kong pour son seul pôle d'excellence scientifique. En 2005, les sommes consacrées aux pôles ont été à 57,5\% consacrées à 14 «pôles mondiaux » ou "à vocation mondiale », alors que les 30 autres projets se sont partagés les 42,5\% restants (Breugnon et al., 2006). Cette opération est pourtant un véritable révélateur des potentialités existantes, comme nous l'avons vu. Or dans ce genre d'opération, il est capital de parvenir à accrocher la dynamique en cours. En effet, au-delà de cette politique, c'est l'organisation de l'espace européen de la recherche et de la haute technologie qui est en jeu. Dans ce genre de situation, où une donnée structurelle de l'organisation du territoire des entreprises est institutionnalisée, il est à craindre que les pôles qui seront laissés de côté ne soient définitivement marginalisés, à la fois parce les choix des entreprises s'effectuent à des moments particuliers et fixent durablement les choses (Fache, 1998, 1999) et parce que les choix institutionnels, lourds et durables, vont alimenter des écarts régionaux pour en faire des données structurelles. La fièvre des technopôles a ouvert une ère des possibles depuis trente ans. Les stratégies de pôles de compétitivité et celles qui suivront risqueraient fort de la clore.

17. Cf. R. Suire (2003) qui fait un rapide historique de la concentration des 300 entreprises de NTIC dans le quartier du Sentier en crise à la fin des années 1990.

18. La PACA fédère ses ressources dans le pôle Solutions Communicantes Sécurisées; Toulouse et Bordeaux intègrent une part de leurs activités réseaux dans le pôle Aéronautique, Espace et Systèmes embarqués; La Bretagne constitue un pôle Image et Réseaux.

19. Chiffres du ministère de l'Économie, cités par L. Chesnais et F. Pijaudier-Cabot (2005). 


\section{Bibliographie}

Benko (G.), 1991. - Géographie des technopôles, Paris, Masson, 224 p.

Bernardy de Sigoyer (M.), Boisgontier (P.), 1988. - Grains de technopole, les micro-entreprises grenobloises et nowveaux espaces productifs, Grenoble, PUG, 258 p.

Bernardy de Sigoyer (M.), Boisgontier (P.), 1996. - La technopole, une certaine idée de la ville, Paris, L'Harmattan, $254 \mathrm{p}$.

Bonneville (M.), 1997. - Lyon, métropole régionale ou euro-cité?, Paris, Anthropos, 202 p.

Breugnon (G.), Gobin (M.), Masseau (P.), Rabiller (J.) (2006). - Les pôles de compétitivité sont-ils crédibles?, monographie de Licence, Nantes, 43 p.

Chesnais (L.), Pijaudier-Cabot (F.), 2005. - « Pôles de compétitivité : de nouveaux projets pour la politique industrielle », Industries, ${ }^{\circ}$ 107, septembre, p. 10-24.

Colletis (G.), Gilly (J.-P.), Pecqueur (B.), Perrat (J.), Zimmerman (J.-B.), 1997. - « Firmes et territoires : entre nomadisme et ancrage territorial ", Espace et Société nº 88/89, p. 115-137.

Decoster (E.), Matteaccioli (A.), Tabaries (M.), 2004. - « Les étapes d'une dynamique de territorialisation : le pôle optique en Île de France », Géographie, Économie et Société, n 4, p. 383-413.

FACHE (J.), 1998. - «Organisation géo-technologique des firmes productrices de biens à haute technologie », $R E R U, \mathrm{n}^{\circ} 1, \mathrm{p} .85-106$.

—, 1999. - «Organisation territoriale des firmes et territoires nationaux », Norois, n 182 , p. 233-255.

—, 2005. - «Atlanpole : la recomposition discrète d'un territoire », Cahiers Nantais, n 62-63, p. 79-88.

Ferguenne (A.), Trimeche (H.), 2005. - « Potentiel scientifique, externalités territoriales et développement des biotechnologies : analyse à partir du cas de Rhône-Alpes », Géographie, Économie et Société, n 4 , p. 405-425.

FerRandon (B.), 2004. - Croissance et Innovation, Cahiers Français, n 323, novembre-décembre, 96 p.

FreEman (D.), 1985. - « The importance of being the first : preemption by early adopters of farming innovations in Kenya », Annals of the Association of American Geographers, p. 117-128

GENET (C.), 1997. - «Quelles conditions pour la formation des biotechnopoles : une étude dynamique », RERU, n 3, p. 405-424.

Grondeau (A.), 2005. - « Le modèle technopolitain en question... : l'exemple comparé de Sophia-Antipolis et de la Silicon Valley», Cahiers Nantais, n 62-63, p. 89-97.

Grossetti (M.), Bes (M.-P.), 2002. - «Effets de proximité : savoirs tacites ou encastrement?», RERU, $\mathrm{n}^{\circ} 5$, p. 777-788.

Jacquet (N.), Darmon (D.), 2005. - Les pôles de compétitivité, le modèle français, Paris, La Documentation Française, 123 p.

Kleinsmager (R.) 1999. - Strasbourg, une ambition européenne, Paris, Anthropos, 216 p.

Leamer (E.), Storper (M.), 2005. - « La géographie économique à l'heure d'Internet », Géographie, Economie et Société, n 4, p. 381-404.

PAillard (S.), 2004. - «Quelles politiques de soutien à la recherche et à l'innovation technologique? », Cahiers Français, novembre-décembre, p. 42-47.

QuÉRÉ (M.), 2002. - «Territoire et gouvernance locale : le cas de Sophia-Antipolis », Géographie, Economie et Société, $\mathrm{n}^{\circ} 2$, p. 225-246.

Suire (R.), 2003. - « Stratégies de localisation des firmes du secteur des TIC : du cyberdistrict au district lisière », Géographie, Économie et Société, n 3-4, p. 379-397.

Source statistique du technopôle

Annuaire Atlanpole 1999, 2001, 2003, 2004, 2005 : [www.atlanpole.fr]

Cet article a été reçu le 26 janvier 2006 et définitivement accepté le 31 août 2006. 
